Original Research Paper

\title{
Ethanol Production by Novel Proline Accumulating Pichia kudriavzevii Mutants Strains Tolerant to High Temperature and Ethanol Stresses
}

\author{
*Rika Indri Astuti, Sena Alifianti, Ratu Nabila Maisyitoh, Nisa Rachmania Mubarik \\ and Anja Meryandini
}

Division of Microbiology, Department of Biology, Faculty of Mathematics and Natural Sciences, Bogor Agricultural University, Bogor 16680, Indonesia

\author{
Article history \\ Received: 7-08-2018 \\ Revised: 1-09-2018 \\ Accepted: 10-09-2018 \\ *Corresponding Author: \\ Rika Indri Astuti \\ Bogor Agricultural University, \\ Bogor, Indonesia \\ Email: rikaindriastuti@apps.ipb.ac.id
}

\begin{abstract}
Accumulation of osmoprotectant molecule, proline, has been reported to induce a stress tolerance phenotype in yeast cells. In this study, two ethanologenic isolates of yeast, Pichia kudriavzevii ( $\mathrm{R}$ and $\mathrm{T}$ ), both capable of using both five and six sugar compounds to produce ethanol, were mutated via ethyl methanesulfonate (EMS) treatment. Prolineaccumulating mutant strains were selected by using prolineanalogue (L-azetidine-2-carboxylic acid). Mutant strains were confirmed to accumulate proline in various level ranging from $11 \%$ to $154 \%$ compared to the wild type cells. Selected mutant strains were more resistant to high ethanol concentration (15\%) and high temperature stress $\left(45^{\circ} \mathrm{C}\right)$ compared to their Wild Type (WT) cells and industrial bioethanol yeast, Saccharomyces cerevisiae. Interestingly, the fermentation rate of isolate R-T1 and T-T2 was higher than its WT, based on quantitation of reducing sugar and ethanol content in both glucose and mixed glucosexylose fermentations. The highest ethanol production was shown by strain R$\mathrm{T} 1(3.3 \mathrm{~g} / 100 \mathrm{~mL})$ that produced $7 \%$ and $20 \%$ higher ethanol compared to its WT in glucose and glucose-xilose as fermentation substrate, respectively. It is worth noting that ethanol production activity of T-T2 was $22 \%$ and $172 \%$ higher than its WT and industrial yeast $S$. cerevisiae, respectively. Our study indicates that proline accumulation in yeast $P$. kudriavzevii may promote ethanol production, especially in mixed substrate fermentations.
\end{abstract}

Keywords: Pichia kudriavzevii, ethyl Methanesulfonate, AZC-Tolerance, Proline, Sugar Mixture Fermentations

\section{Introduction}

Yeasts that belong to the genera of Pichia have been known for their capability of using both hexose and pentose sugars as substrates for bioethanol productions (Mussatto et al., 2012). On the other hand, S. cerevisiae, the commonly used industrial yeast, is unable to use pentose as a substrate, thus suggesting superiority of Pichia in its application for the 2nd generation of bioethanol productions (Radecka et al., 2015). Microbe used in fermentation is one of the limiting factors for a maximum production of bioethanol. During fermentation, yeasts are exposed to various environmental stresses, including an elevated temperature, osmotic pressure and high ethanol stresses, causing depletion upon ethanol production (Ansanay-Galeote et al., 2001). Thus, for more effective and valuable ethanol production, yeast strain developments are needed, particularly to construct stress tolerance-mutant strains.

Various approaches have been employed to construct mutant yeast capable of combating fermentation-related stresses. In $S$. cerevisiae, modifications of proline metabolism pathway results in mutant strains with enhanced stress-tolerant phenotypes against high temperature stresses, ethanol, freeze drying, dehydration, high salinity (Sekine et al., 2007; Sasano et al., 2012a). For instance, a proline accumulating mutant strain has been successfully constructed through putl-gene deletion (Takagi et al., 2000). Put1, a proline oxidase enzyme, is responsible for converting proline to pyrroline-5-carboxylate or glutamic-gammasemialdehyde (Morita et al., 2003). Furthermore, two various amino acid substitutions of $\gamma$-glutamyl kinase (PRO1), Asp154Asn and Ile150Thr, significantly 
enhance intracellular proline content in $S$. cerevisiae, through desensitization of feedback inhibition of that PRO1 activity (Tatehashi and Takagi, 2013). The amino acid substitution was constructed formerly through random mutagenesis by ethyl methane sulfanate treatment and mutant selection via AZC tolerance assay. The toxicity of AZC can be tolerated through intracellular proline accumulation (Shichiri et al., 2001). Proline has been known for its biological and biotechnological purposes (Sasano et al., 2012b). Indeed, proline plays an important role in maintaining the stability of proteins and membrane structure following stress exposure. In addition, proline has also been reported to inhibit the development of protein aggregate and to reduce reactivity of radical molecules (Takagi et al., 2008).

Development of Pichia-mediated bioethanol production has been reported. Both physiological and genetic engineering pproaches have been employed to enhance the capability of yeast to ferment sugars. For instance, supplementation of $\mathrm{CaCO}_{3}$ and $\mathrm{CaCl}_{2}$ in sugar mixture (glucose and xylose) fermentation could increase ethanol production by Pichia for nearly 200\% (Okonkwo et al., 2016). Spontaneous mutation of Pichia stipitis NRRL Y-7124 has also been employed to construct acetic acid tolerant strains, which is important for fermentation using lignocellulose hidrolysate as substrate (Nigam, 2001). To date, development of Pichia yeast strain through modifications of proline metabolism pathway has never been reported yet. Thus in this study we constructed proline-accumulating Pichia mutant strains and investigated its phenotype following stress exposures. Two isolates of $P$. kudriavzevii were used in this study. We managed to employ random mutagenesis to construct proline-accumulating strains. Interestingly, the viability of proline accumulating mutant strains under stress exposures was higher compared to that of wild type strains. Further analysis on the ethanol productions showed that mutant strains were able to produce higher ethanol yield in sugar mixture substrate, compared to wild type strains.

\section{Materials and Methods}

\section{Strains and Culture Media}

Two isolates of Pichia kudriavzevii (isolate $\mathrm{R}$ and $\mathrm{T}$ ) formerly isolated from rotten tropical fruits were routinely maintained in a Yeast Peptone Dextrose medium. Both yeast isolates were used as our former study revealed their capability to ferment glucose and xylose in oxidative-fermentative medium.

\section{Random Mutagenesis}

Construction of mutant was performed as described earlier (Takagi et al., 1997). Mutations were induced by treatment of $4 \%$ with ethyl methane sulfonate in each mid-log phase $P$. kudriavzevii culture for five hours. The culture was then serially diluted and the mutagenized cells were then cultured in Synthetic Dextrose (SD) medium $(0.17 \%$ Yeast nitrogen base without amino acids and ammonium sulfate, $2 \%$ glucose and $0.1 \%$ allantoin) containing $5 \mathrm{mg} / \mathrm{ml}$ proline analogues, L-Azetidine-2carboxylic acid (AZC, Sigma). After incubation at $30^{\circ} \mathrm{C}$ for four days, the resulting colonies were picked up and spread to the SD medium with increased AZC concentrations $(7 \mathrm{mg} / \mathrm{ml})$. The growing colonies were then once again spread in higher AZC treatment $(10 \mathrm{mg} / \mathrm{ml})$. Mutagenized yeast colonies with a larger diameter than other growing colonies were chosen for further analysis.

\section{Proline Content Analysis}

Selected mutant colonies were cultured in Yeast Peptone Dextrose (YPD) medium for $24 \mathrm{~h}$ to mid log phase in $5 \mathrm{ml}$ culture. Collected cells were then washed with $0.9 \% \mathrm{NaCl}$ twice and re-suspended in $0.5 \mathrm{~mL}$ distilled water. Cells were then incubated in boiling water for $10 \mathrm{~min}$ to extract cells lysate. Cell suspensions were then centrifuged (5 min, $15.000 \mathrm{rpm})$. The collected supernatants were then concentrated with evaporator to 100 $\mu \mathrm{L}$. The Intracellular content of the proline was measured by amino acid analyzer (L-8500A-Hitachi, Japan).

\section{Stress Tolerance Assay}

This assay was performed by a simple spot assay method in media containing different stress conditions. Both mutant and wild type yeast strains were cultured in a YPD medium and incubated for $24 \mathrm{~h}$ at room temperature as the main culture. Serial dilution was then performed. About $3 \mu \mathrm{L}$ of culture from each dilution was spotted on top of the YPD media containing various ethanol concentrations $(5 \%, 10 \%, 12 \%$ and $15 \%)$. For high temperature stress, the YPD medium containing spotted culture was incubated in 37,40 and $45^{\circ} \mathrm{C}$. Plates were incubated for 3 days.

\section{Reducing Sugar Content Measurement}

Selected mutant colonies were evaluated for the sugar consumption rate during fermentation. Yeast colonies of both mutant and wild type strains were cultured in a fermentation medium containing different carbon source, $2 \%$ glucose and xylose. Reduced sugar content was calculated by using the 3,5-Dinitrosalicylic acid (DNS) method as described earlier (Miller, 1959). Each independent glucose and xylose standard curve was used as standards. For sugar mixture fermentations, a glucose standard curve was used to calculate the reducing sugar content.

\section{Quantitation of Ethanol Production}

The fermentation culture of yeast colonies of both mutant and wild type strains was prepared by using a 
fermentation medium containing either glucose or xylose only and a glucose-xylose mix (50\% each). The composition of the fermentation medium, which was YP medium, contained $1 \%$ yeast extract and $2 \%$ peptone with addition of $20 \mathrm{~g} / \mathrm{L}$ sugar (glucose or xylose). Ethanol content was measured by piknometer following 6, 30 and $48 \mathrm{~h}$ of fermentation. Ethanol content was measured using formula as described by Avicor et al. (2015).

\section{Results}

\section{Construction of Proline Accumulating Mutant Strains}

Random mutagenesis through EMS treatment resulted in numerous mutant colonies. However, the only mutants that showed viability better than the wild type cells in SD medium containing $7 \mathrm{mg} / \mathrm{ml}$ and $10 \mathrm{mg} / \mathrm{ml}$
AZC (Fig. 1) were selected to use for further analysis. It is worth noting that wild type strains showed severe growth under AZC-mediated proteotoxic stress. Thus emerging the possibility of proline accumulation in the mutant cells. Interestingly, the intracellular proline content of $P$. kudriavzevii isolate R- derived mutants (RT0 and R-T1) to its wild type was not significantly different, yet resulted in essentially different phenotype in corresponding to the capability of mutants to grow in AZC stress-medium. A contrarily phenomenon was found in R-T3 which had the lowest proline content of all mutant tested (18.3nmol/ml) (Fig. 2A); however, the viability of this mutant strain in AZC containing medium was higher than its WT (Fig. 1). On the other hand, all of the mutant strains of that isolate $\mathrm{T}$ showed higher intracellular proline content compared to their WT strains (Fig. 2B).
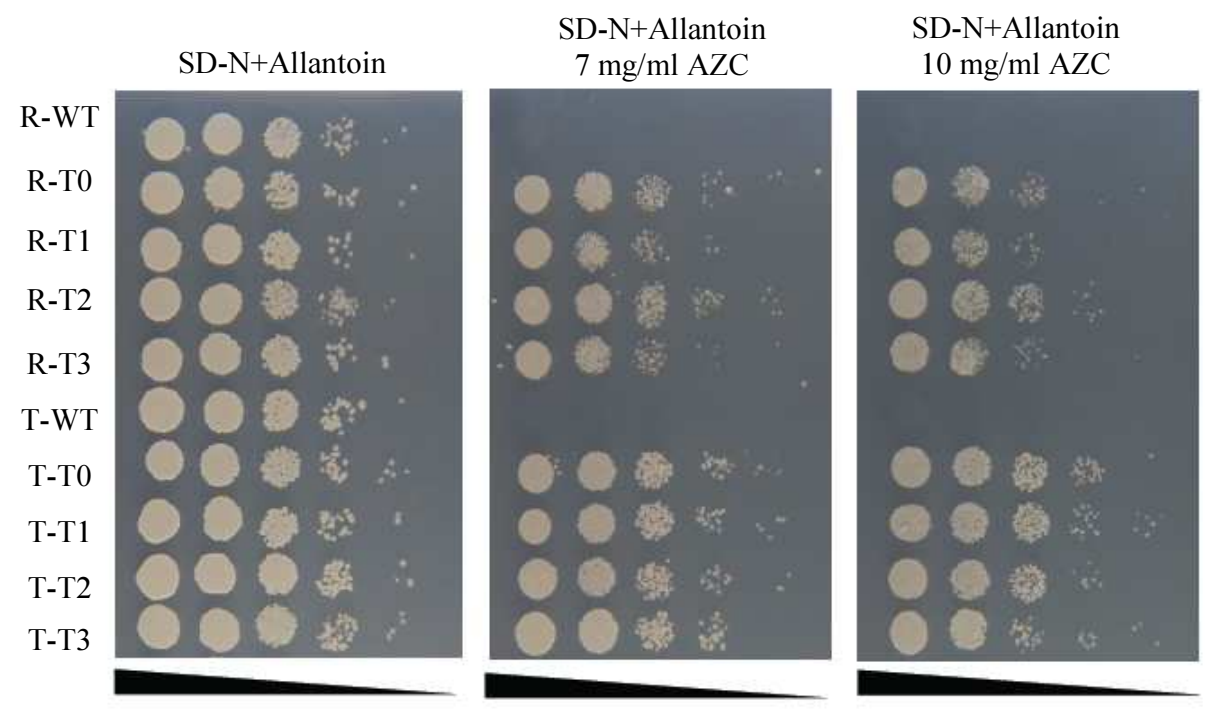

Fig. 1: Spot test assay of $P$. kudriavzevii mutant and wild type strains in medium contaning proline analogue, AZC. RWT: isolate Rwild type; T-WT: isolate T-wild type; T1-T3: EMS-derived mutant strains of each isolate R and T. Colonies were grown for 2 days in $30^{\circ} \mathrm{C}$

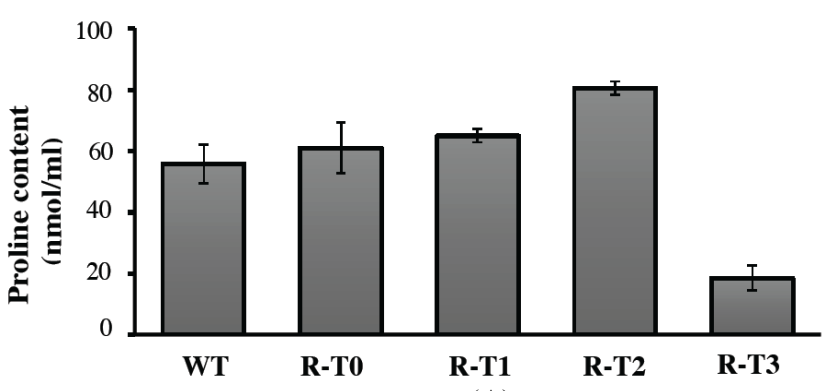

(A)

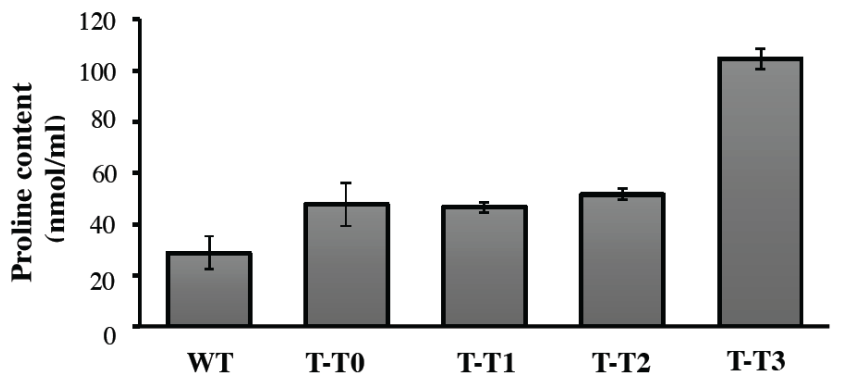

(B)

Fig. 2: The intracellular proline content of wild type and mutant strains of $P$. kudriavzevii (A) isolate R and (B) isolate T. Each yeast strains were grown in SD medium for $24 \mathrm{~h}$ until mid log phase, $5 \mathrm{mg} / \mathrm{ml} \mathrm{AZC}$ was then subjected to the culture for 3 h. Cells were then harvested and extracted to produce cells lysate. Intracellular proline from cell lysate was measured three times by Amino Acid Analyzer 


\section{Stress Tolerance Assays}

As expected, mutant strains were more tolerant to high temperature stress compared to their wild type strains (Fig. 3). Wild type strains of both isolate $\mathrm{R}$ and $\mathrm{T}$ exhibited severe growth following high temperature stress conditions (Fig. 3). In high ethanol stress (15\%), mutant R-T1 and R-T2 as well as T-T2 showed a higher survival rate than their respective wild type strains. Interestingly, mutant strains that exhibited high temperature stress tolerance were not essentially resistant to ethanol stress treatment, as shown by most of the isolate T-derived mutants strains. These results indicate that the EMS-based random mutagenesis likely mediates the stress tolerance mechanism in $P$. kudriavzevii, essential for combating high temperature stress conditions but does not protect against ethanol stress. Thus, further analysis of high temperature stress tolerance pathways of those mutant strains is needed. Based on the spot test assay, one mutant strain from each wild type isolate which showed stress tolerance phenotype were chosen for further analysis, including RT1 and T-T2.

\section{Reducing Sugar Measurement}

The reducing sugar content was measured in a fermentation culture with glucose, xylose and glucosexylose sugar mixtures as substrate (Fig. 4). Mutant strains R-T1 and T-T2 showed relatively similar patterns in glucose consumption to their wild type strains (Fig. 4A-B). Interestingly, mutant strains were capable of using glucose in a relatively higher rate compared to $S$. cerevisiae, the commonly used industrial yeast (Fig. 4A-D). Both mutant R-T1 and TT2 were capable of effectively using both glucose and xylose within $48 \mathrm{~h}$ of sugar mixture fermentation, compared to $S$. cerevisiae (Fig. 4C-D). Indeed, the content of reducing sugar of $S$. cerevisiae culture in the glucose-xylose mixture medium remain stable after $30 \mathrm{~h}$ on incubation, while in $P$. kudriavzevii of both WT and mutants strains continued to decrease in a time dependent manner (Fig. 4C-D). For xylose containing medium, the reducing sugar content within $48 \mathrm{~h}$ culture remained similar between WT and mutant strains (Fig. 4E-F).

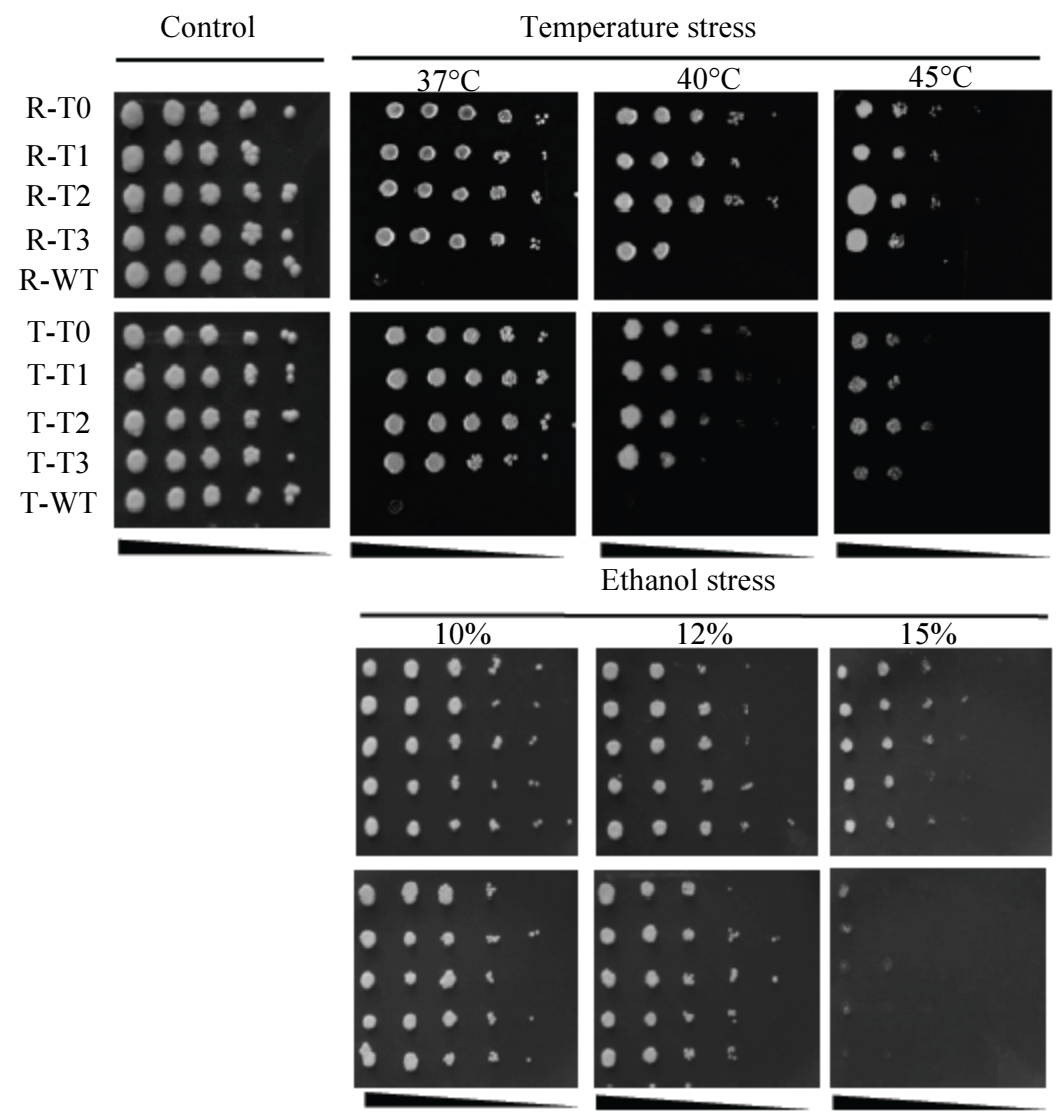

Fig. 3: Spot test assay of $P$. kudriavzevii mutant and wild type strains in high temperature stress $\left(37^{\circ} \mathrm{C}, 40^{\circ} \mathrm{C}, 45^{\circ} \mathrm{C}\right)$. and ethanol stress conditions $\left(10,12\right.$ and $15 \%(\mathrm{v} / \mathrm{v})$ ethanol). Incubation at $30^{\circ} \mathrm{C}$ in YPD medium was used as control. All treatment was incubated for three days. R-WT: isolate R- wild type; T-WT: isolate T-wild type; T1-T3: EMS-derived mutant strains of each isolate $\mathrm{R}$ and $\mathrm{T}$ 

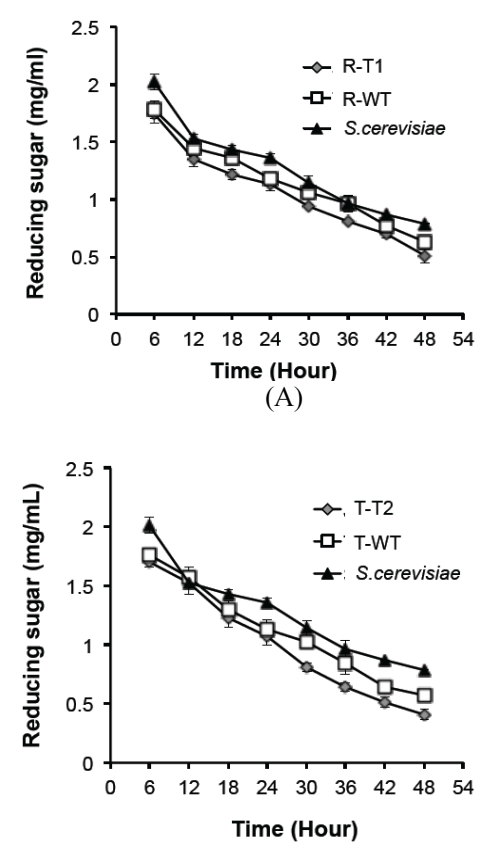

(B)

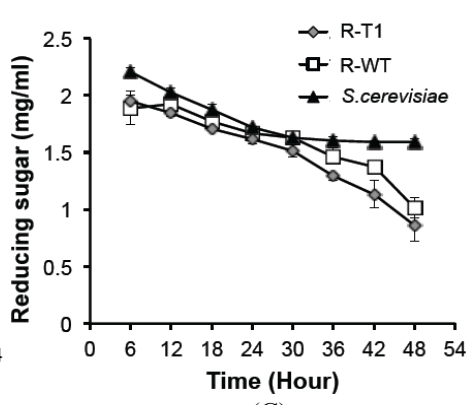

(C)

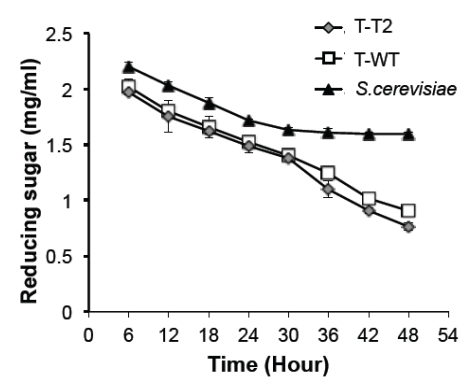

(D)

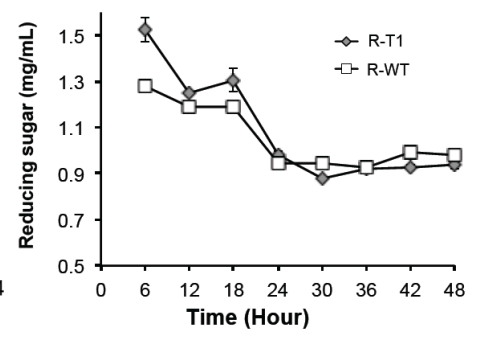

(E)

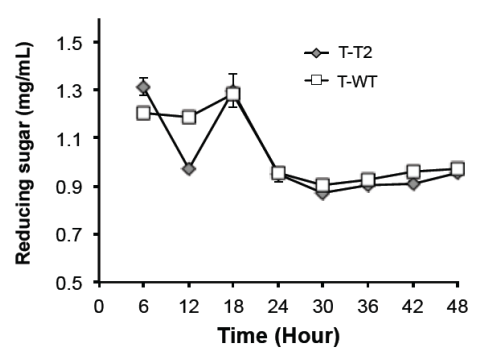

(F)

Fig. 4: Changes in reducing sugar content during fermentation using various substrate (A-B) glucose, (C-D) sugar mixture glucose:xylose and (E-F) xylose by each mutant R-T1 and T-T2. Reducing sugar content was measured by using DNS method. Wild type strain (R and T) and $S$. cerevisiae were used as control.

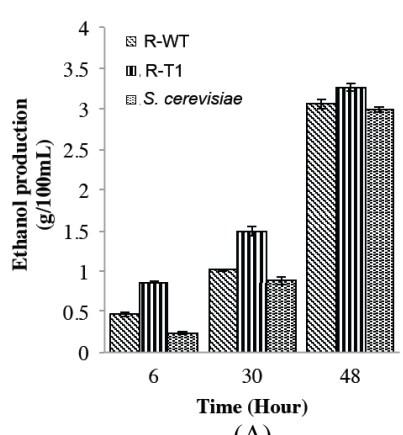

(A)

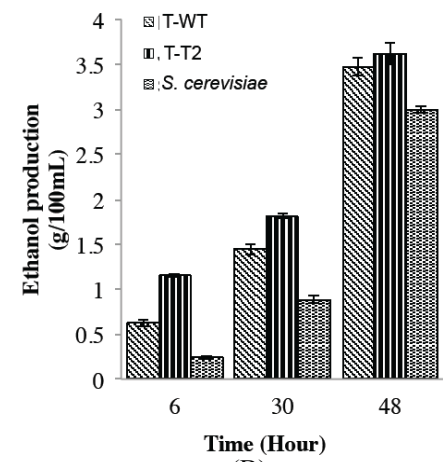

(B)

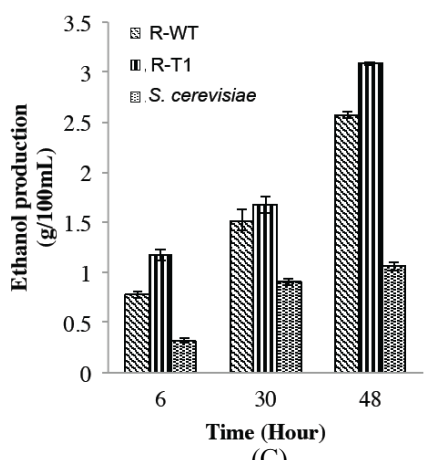

(C)

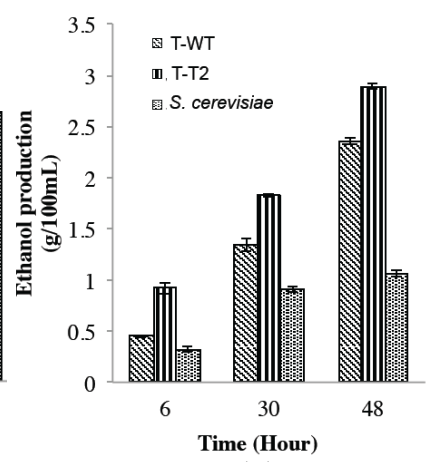

(D)

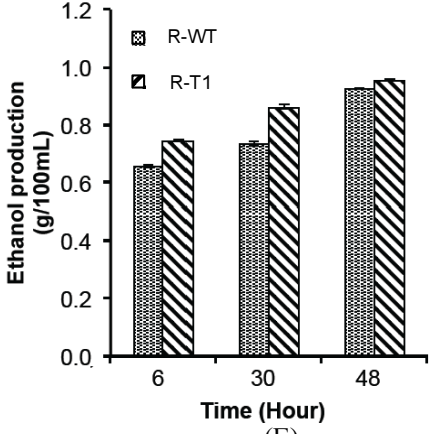

(E)

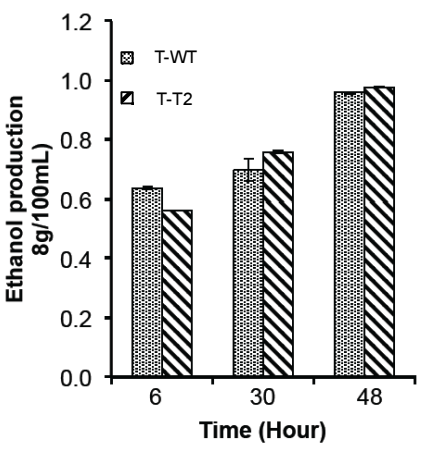

(F)

Fig. 5: Ethanol production by mutant strain R-T1 and T-T2 compared to WT strain and S. cerevisiae in different carbon source (A-B) glucose, (C-D) sugar mixture glucose:xylose and (E-F) xylose. Fermentation was conducted for $48 \mathrm{~h}$. Wild type strain and $S$. cerevisiae were used as control 


\section{Ethanol Production}

Production of ethanol in the glucose of mutant strain R-T1 and T-T2 slightly increased as compared to corresponding wild types (Fig. 5A-B). For instance, RT1 produced $3.3 \mathrm{~g} / 100 \mathrm{~mL}$ ethanol which was $7 \%$ and $9 \%$ higher than its wild type strain and $S$. cerevisiae, respectively (Fig. 5A). On the other hand, mutant T-T2 produced an $11 \%$ higher ethanol yield compared to R-T1 mutant after $48 \mathrm{~h}$ of the fermentation period and approximately $21 \%$ higher ethanol production than $S$. cerevisiae (Fig. 5B). This suggests its strong potential as a fermentation agent among mutant strain tested.

A slight decrease in ethanol production was found in mutant strains when using sugar mixture (xyloseglucose) as a substrate compared to glucose fermentation (Fig. 5CD). Here, we found that at most $15 \%$ lower ethanol production was found in sugar mixture fermentation than glucose fermentation of both mutant strains. However, mutant R-T1 and T-T2 were capable of producing higher ethanol yields than their respective WT strains and $S$. cerevisiae in sugar mixture fermentations (Fig. 5C-D). Indeed, production of ethanol by R-T1 was $20 \%$ and $190 \%$ higher than its WT strain and S. cerevisiae, respectively (Fig. 5C). A relatively similar fermentation rate was also shown by mutant TT2 which was capable of producing $22 \%$ and $172 \%$ higher ethanol content compared to its WT and S. cerevisiae after $48 \mathrm{~h}$ of the fermentation period (Fig. 5D). We noticed that production of ethanol by both mutant strains R-T1 and T-2 was not significantly different when using sugar mixture as substrate. It is worth noting that there was $50 \%$ depletion of ethanol production by $S$. cerevisiae in sugar mixture fermentation compared to glucose fermentation.

Ethanol production in xylose as the main carbon source resulted a relatively similar value between wild type and mutant strains of $P$. kudriavzevii (Fig. 5E-F). Interestingly, $60 \%$ depletion of ethanol production was found in xylose fermentation compared to that of glucose fermentation. This suggests that glucose may likely serve as the preferred carbon source for $P$. kudriavzevii's mutant and wild type strains in producing ethanol.

\section{Discussion}

During fermentation, yeasts are exposed to various types of fermentation-related stresses, including high temperature, osmotic, oxidative and ethanol stress. Thus, observation and discovery of novel stress tolerance of yeast from potential samples, such as fermented food and beverages as well as agricultural waste management system,s are currently conducted for further application in the bioethanol industry (Steensels and Verstrepen, 2014; Zabed et al., 2017). Modifications upon yeast metabolic pathways to optimize ethanol production is gaining significant interest worldwide (Alper et al.,
2006; Ekberg et al., 2013). Various studies regarding genetic and physiological engineering upon yeast isolates as a fermentation agent has been conducted (Zhao and Bai, 2009; Ha et al., 2011; Lam et al., 2014; Harner et al., 2015). Advanced research in omics also provide significant development in yeast engineering to support an efficient ethanol production in various types of substrates (Marks et al., 2008). Pichia is one of the major interests of biofuel-related research, since this yeast genera can deliver both hexose and pentose sugar fermentations (Steensels and Verstrepen, 2014). This suggests its potential application in 2nd bioethanol production.

In this study, we objected to modify the proline metabolic pathway of non-conventional yeast $P$. kudriavzevii to enhance stress tolerance phenotype against high temperature and ethanol stresses. Random mutagenesis was applied to construct Pichia mutant strains since this genera of yeast has not been widely explored particularly its genetic properties of stress tolerance mechanisms. Thus random modifications upon genetic sequence via EMS was used to construct the targeted mutant instead of target mutagenesis.

Proline accumulating strains of $P$. kudriavzevii was constructed by random mutagenesis. In this study, the proline accumulating mutant strains derived from directed evolution in AZC treatment showed potential characters as fermentation agent. For instance, both mutant R-T1 and T-T2 mutants were able to survive in $45^{\circ} \mathrm{C}$ and $15 \%$ ethanol stresses. Following those potential characters, the ethanol production of mutant in glucose and sugar mixture fermentation was also higher than its wild type and industrial yeast strains, $S$. cerevisiae. This suggests that genetic changes through directed evolution is involved in modulating ethanolsynthetic pathway. Previous studies have confirmed that proline may act as a cytoprotective molecule that increases cells' viability during fermentation and significantly increases fermentation yield. Both exogenous and genetic modifications-derived proline accumulation may induce a stress tolerance mechanism in yeast (Takagi et al., 2005; Kaino et al., 2008; Sasano et al., 2012a; 2012b).

Interestingly, mutant strains of $P$. kudriavzevii isolate $\mathrm{R}$ and $\mathrm{T}$ both showed high AZC-resistant phenotypes against $10 \mathrm{mg} / \mathrm{ml} \mathrm{AZC}$. It is worth noting that such high AZC-tolerance phenotypes exhibited by yeast $P$. kudriavzevii mutant strains is quite remarkable, since most of the yeast cells could tolerate relatively lower amount of AZC $(0.5-2 \mathrm{mg} / \mathrm{ml})$. Mutant R-T3 showed distinct character to the other mutant tested, since its intracellular proline content was significantly lower than its WT strains, yet showed an AZC-resistant phenotype. It is likely that an AZC-tolerance mechanism in R-T3 may occur in a proline-independent pathway. A previous study had reported that yeast may remain viable following exposure of AZC-proteotoxic stresses by 
inducing ubiquiting ligase Rsp5 activity (Hiraishi et al., 2006). Mutation of the general amino acid permeaseencoding gene, GAP1, in $S$. cerevisiae also induced an AZC-tolerant phenotype (Haitani et al., 2009).

It is worth noting that, in sugar mixture fermentations, mutant strains, primarily T-T2, produced lower ethanol concentrations than in glucose fermentations. Such phenomenon had been previously reported as "glucose repression". Shi et al. (2014) also reported that the production of ethanol of mutant $P$. stipitis TJ2-3 was relatively similar to that WT cells in mixture sugar fermentations. The presence of glucose will repress the fermentation of xylose so that when the glucose is completely utilized, ethanol can no longer be produced. However, considering the high capability of mutant R-T1 and T-T2 of producing at most $3 \mathrm{~g} / 100 \mathrm{ml}$ ethanol, it is suggested that mutant strains were capable of employing their metabolism pathways to efficiently use xylose in the same manner to that of glucose utilization. Indeed, yeast genera of Pichia has been reported to possess pentose phosphate pathways, in addition to glycolysis, that serve as the main carbon utilization pathway when yeast cells are cultivated in a pentose sugar medium (Jeffries et al., 2007; Agbogbo and Coward-Kelly, 2008).

In media with xylose as the main carbon source, the efficiency of ethanol production is lower than glucosecontaining media. A previous study had reported that a protein transporter responsible for xylose uptake in Pichia stipitis (Sut1) had lower transport capacity than that $S$. cerevisiae's xylose transporter (Gfxl) (Runquist et al., 2010). Thus it is likely that the xylose transport rate may occur in a slow manner that leads to the lower xylosedependent ethanol production as shown by both mutant and WT strains.

\section{Conclusion}

From our study, it is suggested that the development of a stress tolerant yeast of $P$. kudriavzevii through mutagenesis may enhance yeast performance in ethanol production. Stress tolerant phenotypes of mutant strains P. kudriavzevii R-T1 and T-T2 may play an important role in mediating ethanol production in a significantly higher yield than its wild type and conventional industrial yeast, $S$. cerevisiae. The ability of mutants to utilize glucose and xylose suggests its future application in 2nd generation of bioethanol productions.

\section{Acknowledgement}

The authors thank to Prof. Hiroshi Takagi of Nara Institute of Science and Technology, Japan for kindly provide $S$. cerevisiae isolate.

\section{Funding Information}

This project was granted by Penelitian Terapan Unggulan Perguruan Tinggi, The Ministry of Research, Technology and Higher Education [1359/IT3.11/PN/2017] to RIA.

\section{Author's Contributions}

Rika Indri Astuti: Had led the project, contributed in the experimental design, data analysis and manuscript writing.

Sena Alifianti: Had contributed in laboratory experiments and data analysis.

Ratu Nabila: Had contributed in laboratory experiments and data analysis.

Nisa Rachmanis Mubarik: Had contributed experimental design and data analysis.

Anja Meryandini: Had contributed experimental design and data analysis.

\section{Ethics}

This article is authentic from authors works. The corresponding author ensures that all of the other authors have read and recognized the manuscript.

\section{References}

Agbogbo, F.K. and G. Coward-Kelly, 2008. Cellulosic ethanol production using the naturally occurring xylose-fermenting yeast, Pichia stipitis. Biotechnol. Lett., 30: 1515-1524. DOI: $10.1007 / \mathrm{s} 10529-008-9728-\mathrm{z}$

Alper, H., J. Moxley, E. Nevoigt, G.R. Fink and G. Stephanopoulos, 2006. Engineering yeast transcription machinery for improved ethanol tolerance and production. Science, 314: 1565-1568. DOI: 10.1126/science.1131969

Ansanay-Galeote, V., B. Blondin, S. Dequin and J.M. Sablayrolles, 2001. Stress effect of ethanol on fermentation kinetics by stationary-phase cells of Saccharomyces cerevisiae. Biotechnol. Lett., 23: 677-681. DOI: 10.1023/A:1010396232420

Ekberg, J., J. Rautio, L. Mattinen, V. Vidgren and J. Londesborough et al., 2013. Adaptive evolution of the lager brewing yeast Saccharomyces pastorianus for improved growth under hyperosmotic conditions and its influence on fermentation performance. FEMS Yeast Res., 13: 335-349. DOI: $\underline{10.1111 / 1567-1364.12038}$

Ha, S.J., J.M. Galazka, S.R. Kim, J.H. Choi and X. Yang et al., 2011. Engineered Saccharomyces cerevisiae capable of simultaneous cellobiose and xylose fermentation. Proceedings National Academy Sci., 108: 504-509. DOI: 10.1073/pnas. 1010456108 
Haitani, Y., M. Nakata, T. Sasaki, A. Uchida and H. Takagi, 2009. Engineering of the yeast ubiquitin ligase Rsp5: isolation of a new variant that induces constitutive inactivation of the general amino acid permease Gap1. FEMS Yeast Res., 9: 73-86. DOI: $10.1111 /$ j.1567-1364.2008.00460.x

Harner, N.K., X. Wen, P.K. Bajwa, G.D. Austin and C.Y. Ho et al., 2015. Genetic improvement of native xylosefermenting yeasts for ethanol production. J. Industrial Microbiology Biotechnol., 42: 1-20. DOI: $10.1007 / \mathrm{s} 10295-014-1535-\mathrm{z}$

Hiraishi, H., M. Mochizuki and H. Takagi, 2006. Enhancement of stress tolerance in Saccharomyces cerevisiae by overexpression of ubiquitin ligase Rsp5 and ubiquitin-conjugating enzymes. Biosci. Biotechnol. Biochem., 70: 2762-2765.

DOI: $10.1271 / \mathrm{bbb} .60250$

Jeffries, T.W., I.V. Grigoriev, J. Grimwood, J.M. Laplaza and A. Aerts et al., 2007. Genome sequence of the lignocellulose-bioconverting and xylosefermenting yeast Pichia stipitis. Nature Biotechnol., 25: 319. DOI: $10.1038 /$ nbt1290

Kaino, T., T. Tateiwa, S. Mizukami-Murata, J. Shima and H. Takagi, 2008. Self-cloning baker's yeasts that accumulate proline enhance freeze tolerance in doughs. Applied Environ. Microbiol., 74: 5845-5849. DOI: 10.1128/AEM.00998-08

Lam, F.H., A. Ghaderi, G.R. Fink and G. Stephanopoulos, 2014. Engineering alcohol tolerance in yeast. Science, 346: 71-75.

DOI: $10.1126 /$ science. 1257859

Marks, V.D., S.J. Ho Sui, D. Erasmus, G.K. Van Der Merwe and J. Brumm et al., 2008. Dynamics of the yeast transcriptome during wine fermentation reveals a novel fermentation stress response. FEMS Yeast Res., 8: 35-52. DOI: $10.1111 / \mathrm{j} .1567-1364.2007 .00338 . x$

Miller, G.L., 1959. Use of dinitrosalicylic acid reagent for determination of reducing sugar. Analytical Chemistry, 31: 426-428.

DOI: $10.1021 / \mathrm{ac} 60147 \mathrm{a} 030$

Morita, Y., S. Nakamori and H. Takagi, 2003. L-Proline accumulation and freeze tolerance in Saccharomyces cerevisiae are caused by a mutation in the PRO1 gene encoding $\gamma$-glutamyl kinase. Appl. Environ. Microbiol, 69: 212-219. DOI: 10.1128/AEM.69.1.212-219.2003

Mussatto, S.I., E.M. Machado, L.M. Carneiro and J.A. Teixeira, 2012. Sugars metabolism and ethanol production by different yeast strains from coffee industry wastes hydrolysates. Applied Energy, 92: 763-768. DOI: 10.1016/j.apenergy.2011.08.020

Nigam, J.N., 2001. Ethanol production from wheat straw hemicellulose hydrolysate by Pichia stipitis. J. Biotech., 87: 17-27.

DOI: $10.1016 / \mathrm{S} 0168-1656(00) 00385-0$
Okonkwo, C.C., M.M. Azam, T.C. Ezeji and N. Qureshi, 2016. Enhancing ethanol production from cellulosic sugars using Scheffersomyces (Pichia) stipitis. Bioprocess. Biosyst. Eng.

DOI: $10.1007 / \mathrm{s} 00449-016-1580-2$

Radecka, D., V. Mukherjee, R.Q. Mateo, M. Stojiljkovic and M.R. Foulquié-Moreno et al., 2015. Looking beyond Saccharomyces: The potential of nonconventional yeast species for desirable traits in bioethanol fermentation. FEMS Yeast Res.

DOI: $10.1093 /$ femsyr/fov053

Runquist, D., B. Hahn-Hägerdal and P. Rådström, 2010. Comparison of heterologous xylose transporters in recombinant Saccharomyces cerevisiae. Biotechnology Biofuels. DOI: 10.1186/1754-6834-3-5

Sasano, Y., Y. Haitani, I. Ohtsu, J. Shima and H. Takagi, 2012a. Proline accumulation in baker's yeast enhances high-sucrose stress tolerance and fermentation ability in sweet dough. Int. J. Food Microbiol., 152: 40-43.

DOI: 10.1016/j.ijfoodmicro.2011.10.004

Sasano, Y., Y. Haitani, K. Hashida, I. Ohtsu and J. Shima et al., 2012b. Simultaneous accumulation of proline and trehalose in industrial baker's yeast enhances fermentation ability in frozen dough. J. Biosci. Bioengin., 113: 592-595. DOI: $10.1016 /$ j.jbiosc.2011.12.018

Sekine, T., A. Kawaguchi, Y. Hamano and H. Takagi, 2007. Desensitization of feedback inhibition of the Saccharomyces cerevisiae $\gamma$-glutamyl kinase enhances proline accumulation and freezing tolerance. Appl. Environ. Microbiol, 73: 4011-4019. DOI: 10.1128/AEM.00730-07

Shi, J., M. Zhang, L. Zhang, P. Wang and L. Jiang et al., 2014. Xylose-fermenting Pichia stipitis by genome shuffling for improved ethanol production. Microbial Biotechnol., 7: 90-99.

DOI: 10.1111/1751-7915.12092

Shichiri, M., C. Hoshikawa, S. Nakamori and H. Takagi, 2001. A novel acetyltransferase found in Saccharomyces cerevisiae $\Sigma 1278$ b that detoxifies a proline analogue, azetidine-2-carboxylic acid. J. Biological Chemistry, 276: 41998-42002.

DOI: $10.1074 /$ jbc.C100487200

Steensels, J. and K.J. Verstrepen, 2014. Taming wild yeast: Potential of conventional and nonconventional yeasts in industrial fermentations. Annual Review Microbiol., 68: 61-80. DOI: 10.1146/annurev-micro-091213-113025

Takagi, H., 2008. Proline as a stress protectant in yeast: physiological functions, metabolic regulations and biotechnological applications. Applied Microbiology Biotechnol., 81: 211. DOI: $10.1007 / \mathrm{s} 00253-008-1698-5$ 
Takagi, H., F. Iwamoto and S. Nakamori, 1997. Isolation of freeze-tolerant laboratory strains of Saccharomyces cerevisiae from prolineanalogueresistant mutants. Applied Microbiology Biotechnol., 47: 405-411. DOI: $10.1007 / \mathrm{s} 002530050948$

Takagi, H., K. Sakai, K. Morida and S. Nakamori, 2000. Proline accumulation by mutation or disruption of the proline oxidase gene improves resistance to freezing and desiccation stresses in Saccharomyces cerevisiae. FEMS Microbiol. Lett., 184: 103-108. DOI: $10.1111 / \mathrm{j} .15746968 .2000 . t b 08998 . \mathrm{x}$

Takagi, H., M. Takaoka, A. Kawaguchi and Y. Kubo, 2005. Effect of L-proline on sake brewing and ethanol stress in Saccharomyces cerevisiae. Applied Environ. Microbiol., 71: 8656-8662.

DOI: 10.1128/AEM.71.12.8656-8662.2005
Tatehashi, Y. and H. Takagi, 2013. Characterization of $\gamma$-glutamyl kinase mutants from Saccharomyces cerevisiae. J. Biosci. Bioengin., 116: 576-579. DOI: 10.1016/j.jbiosc.2013.05.018

Zabed, H., J.N. Sahu, A. Suely, A.N. Boyce and G. Faruq, 2017. Bioethanol production from renewable sources: Current perspectives and technological progress. Renewable Sustainable Energy Rev. DOI: 10.1016/j.rser.2016.12.076

Zhao, X.Q. and F.W. Bai, 2009. Mechanisms of yeast stress tolerance and its manipulation for efficient fuel ethanol production. J. Biotechnol., 144: 23-30. DOI: $10.1016 /$ j.jbiotec.2009.05.001 